\title{
The Cognitive Difference of Visual and Imaged Tactile Sense of Product Forms
}

\author{
Mei-Ting $\operatorname{Lin}^{1}$, Jui-Ping $\mathrm{Ma}^{1}$, and Chih-Long $\operatorname{Lin}^{2}$ \\ ${ }^{1}$ Graduate School of Creative Industry Design, \\ National Taiwan University of Art, Taipei, Taiwan \\ ${ }^{2}$ Crafts \& Design Department, National Taiwan University of Art, Taipei, Taiwan \\ gua_gua@mail2000.com.tw, artma2010@gmail.com, CL.Lin@ntua.edu.tw
}

\begin{abstract}
Product morphology has affected the diversity of consumers' preference. When consumers obtain the product image through perceptual organs, it is possible for the visual sense to replace other senses and generate synesthesia. This study is intended to explore: 1. whether if the visual sense and imagined tactile sense of product forms are consistent in preference; and 2. whether if the cognition preference of the four products with different figure is consistent when viewing the objects simultaneously and individually. The experiment designed four cups with different forms and separately processed a two-phase test of the visual and imagined tactile senses. The analysis result shows that visual and imagined tactile sense possesses slight consistency with product preference, which both reached significant level. Therefore, the preference results of most test subjects in both visual and imaginary tactile sense achieved consistency. Among the four cups, Cup 2 revealed far lower score in the visual sense than in the tactile sense within the pleasure degree, while Cup 3 shown far greater visual points than tactile. The results of experimenting the four cups with different forms yet with same color and material, did not agree with our hypothesis, the anticipation to see and the anticipation to touch were in inverse proportion. The outcome indicated that creative design products via the consumer's view may emerge cognitive difference. Thus, the results of this study can provide products which are in development a better understanding of consumer perception and a foundation for future studies.
\end{abstract}

Keywords: Product Form, visual, imagined tactile sense, cognitive differences.

\section{Introduction}

The cultural creative industry has rise in recent years, "the necessity of to cultural product development is to improve the quality of life and social and cultural levels, thus, design is no longer the pursuit for technical development and beautiful forms, but to focus more on cultural heritage and maintenance" [4]. A designer incorporates images into products with the employment of one feature and collocating with other kinds of features to produce his/her creations based on the needs, feelings, and ideas of people. However, the perceptual capability to images varies from person to person, therefore, designers is required to have a good interest and idea of how images, forms, 
and the characteristics of forms are related when the products employed as a media to convey the imagery [7]. Through consumers' concern, products create semantics, the conveyance of the semantics direct response of the products, which includes expression of preference. Semantics is generated through sensory organs, the senses of sight and touch are the cognitive interface of human's primary perception towards products, thus, the Drink Up cup series the from JIA Inc., was selected as test samples to acquire the following two goals: 1 . whether if the visual sense and imagined tactile sense of product forms are consistent in preference; and 2. whether if the cognition preference of the four products with different figure is consistent when viewing the objects simultaneously and individually. The Drink Up series consists of four cups with various forms designed for four family members or friends so that each person can recognize his/her cup without confusion. With such interesting creativity, a test was conducted to find out whether if intention of different forms can really meet the expectations of general consumers.

\section{Product Image}

The visual sense has a dominance trait towards the complex sensory perception of vision and touch and is often a substitute for the tactile sense. From an application level, people in general rely more on the visual sense in perceiving the image of a product, which explains the possibility that complex sensory perceptions are replaced by the visual sense in the same context [2]. Perception is the main foundation for people to transform the product form into image. In addition to acquiring perceptual experience from the stimulation of sensory physiology functions, it can also be obtained through a person's subjective interpretation of stimulations, which classified as sophisticated psychological activity, a few psychological factors may affect the perceptual experience [6]. The activity of forms starts with conveying the expression of an idea and ends with the perception of understanding, the core of the two activities resides in expression and communication. Creative activities originate from the dialogue between the thoughts of the creator, thus, communication consists of not only the outward conveyance of the creator's ideas and the interaction between thoughts within, but also the internal feelings, transformation, sharing, and propagation of the audience [1].Image is a psychological feature, it consist subjective experience to a certain level and is also the reproduction of perceptual experience - creating associative thinking via characteristics expressed by the conceptual meanings of an object through a series of psychological processes consisting perceptual feelings, perceptions, and cognition [3]. The image cognition of product is correlated with the life experience and cultural background of the user, which is classified as the emphasis of perceptual experience in the psychological process; associative thinking includes "visual," "tactile", and "manipulative" [5]. 


\section{$3 \quad$ Method}

There were 81 test subjects selected as valid samples, 23 males and 58 females. Four cups with identical material and color yet different forms (see Fig.1) were processed in a four stage questionnaire.

In Stage 1, the test subjects were given 20 seconds to "visually see" the forms of the four cups simultaneously and asked to provide their order of preference in the most intuitive manner.

In Stage 2, the test subjects were allowed to visually see the four cups individually and provide their preference ratings on a 10-point scale.

In Stage 3, the test subjects were given 20 seconds to "imagined tactile sense" simultaneously and asked to provide their order of preference in the most intuitive manner. In Stage 4, the test subjects were allowed to imaginarily touch the four cups individually and provide their preference ratings on a 10-point scale.

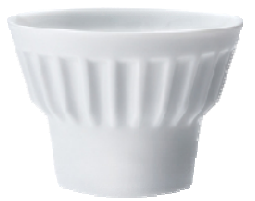

Cup 1

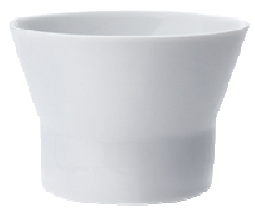

Cup 2

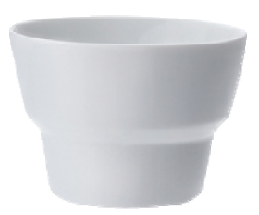

Cup 3

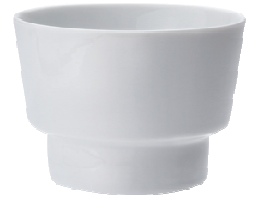

Cup 4

Fig. 1. The four sample items were used in this study. The order from left to right is Cup 1, Cup 2, Cup 3 and Cup 4.

\section{Data Analysis}

The result analysis was conducted in 3 phases with 81 valid questionnaires, analysis information is shown below:

In Phase 1, the analysis focused on the 1 to 4 preference ranking of the "visual sense" tests on the four cups at the same time and the 1 to 4 preference ratings on the four cups of the "imagined tactile sense" tests to see if there is any consistency in these two stages.

In Phase 2, the analysis paid individual attention to the preference ratings from 1 to 10 points on the four cups in the "visual sense" tests and the preference ratings from 1 to 10 points on the four cups in the "imagined tactile sense" tests to see if there is any consistency in these two stages.

In Phase 3, the analysis involves consistency analysis between the simultaneous ranking and individual scores in Stage 1 and 2's "visual sense" tests with the simultaneous ranking and individual scores in Stage 3's "imagined tactile sense" tests. The relation among these 3 phases is shown in Fig. 2. 


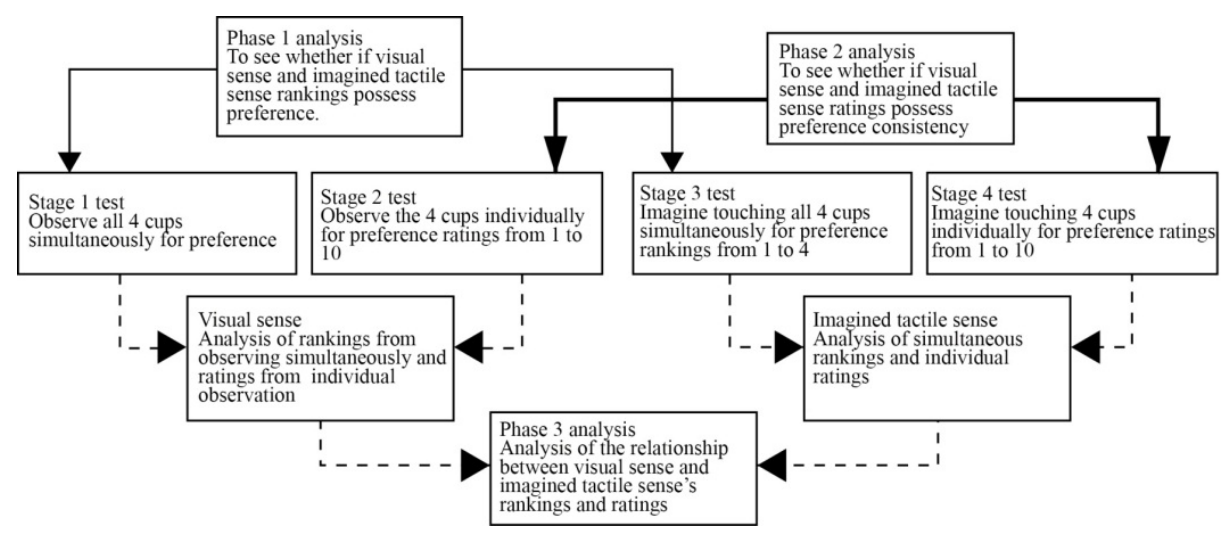

Fig. 2. Analysis diagram

\subsection{Analysis of Preference for Visual and Imagined Tactile Senses}

Phase 1 analysis: the results of rankings given in "visual sense" test of Stage 1 and "imagined tactile sense" of Stage 3 were then processed the aggregation of the individual rating frequency to verify the hypothesis of consistency between visual senses and imagined tactile senses. In both stages of the test, the examinees were allowed to view the four cups simultaneously and write down the rankings for each cup based on their preference. Figures 3 are the quantity distribution outcome of the visual sense test preference ranking and imaged tactile sense test rating of the four cups. The result reveals that cups 1 and 4 possess preference consistency between visual and imagined tactile senses; as for cups 2 and 3, the quantity outcome which ranked first place showed difference in the preference distribution of visual and imagined tactile senses. Only 15 test subjects gave first place to cup 2 regarding visual preference, whereas 29 rated first place in imagined tactile sense. For the quantity distribution of the second to fourth places, consistent decline was found in range of both visual and imagined tactile senses, indicating that the test subjects expected more tactile preference from cup 2 than in visual preference. For cup 3, thirty three test subjects gave first place in visual preference, while only 16 gave tactile preference first place. The consistent decline of the visual and imagined tactile sense preference range indicates that cup 3 provided test subjects with greater visual pleasure than tactile anticipation. One interesting finding is that of the four cups, the form difference between cups 2 and 3 was the smallest, yet the degree of difference after analyzing was actually greater than the other two cups.

Phase 2 analysis: a cross-reference was processed to analyze the individually examined preference results obtained from the "visual sense" test of Stage 2 and "imagined tactile sense" test of stage 4. It was assumed that in Phase 2 analysis that the preference should be consistent no matter if the four cups were observed collectively or individually, therefore, the result should correspond to the outcome of 1 analysis. 

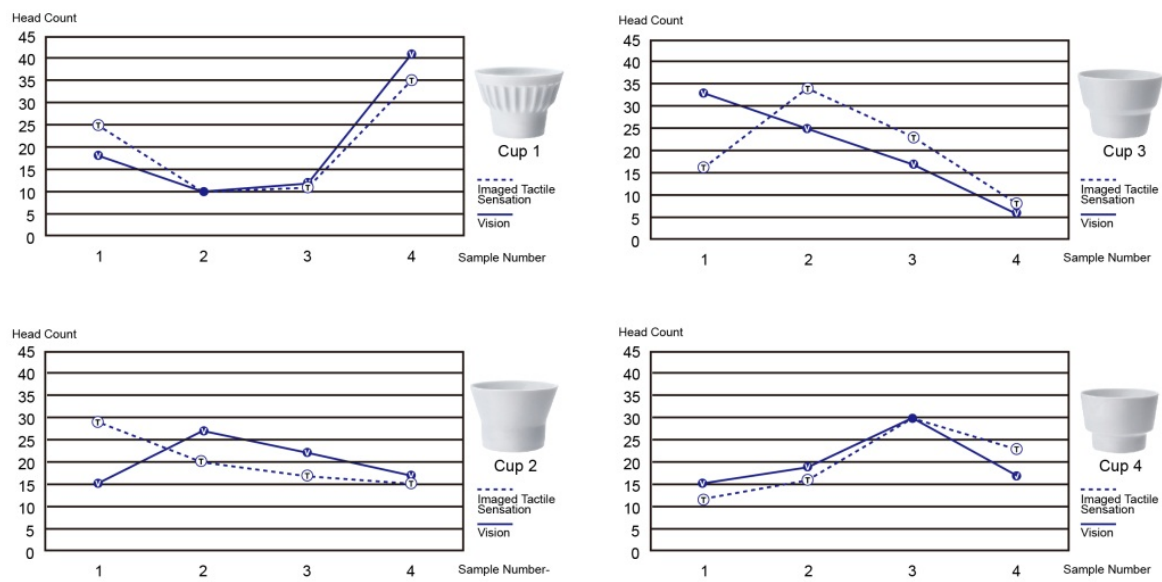

Fig. 3. The quantity distribution outcome of the visual sense test preference ranking and imaged tactile sense test rating of the four cup

During the test, the examinees were allowed to see only one cup at a time before rating the preference of the cup on a 1-10 point scale, 1 being the lowest and 10 being the highest. The Stage 2 "visual sense" test and Stage 4 "imagined tactile sense" test were then conducted separately, the ratings for each of the cups obtained from the two stages were added together (see Table 1). After acquiring the aggregation, the total of each cup was analyzed by the One-way ANOVA analysis to determine an F value of 0.0171 . The tests were conducted to see whether there is difference between the test subject's visual and imagined tactile senses, outcome indicates preference consistency for all four cups, as shown in Table 2. The visual senses and imagined tactile sense rating for each was then analyzed by a two-tailed test, which obtained the results as followed: $.475^{* *}$ for cup $1, .386^{* *}$ for cup $2, .462 * *$ for cup 3 , and $.485^{* *}$ for cup 4 (see Table 3). From the above data, we can realize that the four cups has both achieved significant level in the visual and imagined tactile senses.

The analysis of the 3 phases mentioned above revealed that the assumption where there should be consistency between visual sense and imagined tactile sense and that there should consistency as well as the preferences consistency for observing simultaneously and individually, however, in this case, the result of the four cups being tested together and tested separately did not correspond. The outcome for testing the cups together indicated that the preference of cup 2 was greater in imagined tactile sense than in visual sense; as for cup 3, the preference was greater in visual sense than in imagined tactile sense. 
Table 1. Visual/tactile sense Table 2. One-way analysis of variance (ANOVA) ratings

\begin{tabular}{|c|c|c|c|c|c|c|c|}
\hline & Vision & Tactile & & SS & $\mathrm{df}$ & MS & $F$ \\
\hline Cup1 & 431 & 442 & $\begin{array}{c}\text { Between } \\
\text { Groups }\end{array}$ & 24.5 & 1 & 24.5 & 0.0171 \\
\hline Cup2 & 479 & 508 & Within & 8569.5 & 6 & 1428.25 & \\
\hline Cup3 & 523 & 503 & Group & & & & \\
\hline Cup4 & 472 & 438 & Total & 8594 & 7 & & \\
\hline
\end{tabular}

Table 3. Correlation derived from two-tailed test

\begin{tabular}{lllll}
\hline & cup1 & cup2 & cup3 & cup4 \\
\hline $\begin{array}{l}\text { visual sense \&Imaged } \\
\text { tactile sense }\end{array}$ & $.475^{* *}$ & $.386^{* *}$ & $.462^{* *}$ & .485 \\
\hline
\end{tabular}

\subsection{Consistency Analysis on Preference of Observing Simultaneously and Individually}

Phase 3 analysis: the sequence from 1 to 4 obtained in the Stage 1 "visual sense" test when looking at the cups together and the ratings from 1 to 10 obtained from Stage 2 test when looking at the cups individually were processed according to rank and aggregate scores separately to calculate the means and standard deviations. The same process was conducted on the sequence from 1 to 4 obtained in the Stage 3 "imagined tactile sense" test when looking at the cups together and the ratings from 1 to $10 \mathrm{ob}-$ tained from Stage 4 test when looking at the cups individually. The results were analyzed to find out whether if there is any preference consistency when looking at the cups simultaneously and individually.

Table 4 contains the analysis data which indicates the standard deviations of the four cups in the "visual sense" test. The standard deviations of the selected first place to fourth place gradually increased, indicating that the degree of dispersion increased according to the preference level ranking from first place to fourth place and that, the fourth place given by test subjects showed the greatest rating difference. No patterns were observed in the standard deviations obtained from the "imagined tactile sense" analysis (see Table 5). As for the "visual sense" test, the ranking of preference in observing simultaneously was consistent with the preference ratings in observing individually, yet there were no preference consistency for "imagined tactile" in neither simultaneous nor individual observations. The only consistency observed from both "visual sense" and "imagined tactile sense" tests was the standard deviation for cup 1, which also rank first place, was all smaller than 1, suggesting that cup 1, ranking first place, shows the most consistency when either the cups are observed together or individually in the "visual sense" and "imagined tactile sense" tests. The visual sense standard deviation diagram is shown in Table 4, while the imagined tactile sense standard deviation diagram is shown Table 5; the data acquired from the two tables suggests the ratings if 
the mean decreased from the first place to fourth place, however, the "imagined tactile sense" revealed greater rating difference than "visual sense."

If we divide the 10-point rating scale into 4 sections with 10 9 points being the highest section, 8 6 being the medium high section, 5 3 points being the medium low section, and $2 \sim 1$ points being the lowest section, via means analysis, we realize that cups which ranked first place in the "visual sense" test, scored an average of 7.72 8.50, with a high-low rating difference of 0.78 , the cups was located in the medium high section and failed to reach the highest section. For cups ranking in second place, the mean ratings were 6.68 6.00 with a high-low rating difference of 0.68 , which also located in the medium high section but in the lower half of the section (7 points). For cups ranking third place, the mean ratings were 5.58 5.00 with a high-low rating difference of 0.58 , which fell into the medium low section. For cups ranking fourth place, mean ratings were 4.24 3.00 with a high-low rating difference of 1.24, which also fell into the medium low section. As for the "imagined tactile sense" test, the cups ranking first place were given a mean ratings of 8.64 6.88 with a high-low rating difference of 1.76, which was located below the in the medium high section and failed to reach the highest section. For cups ranking second place, the mean ratings were 6.50 6.05 with a highlow rating difference of 0.45 , which was located in the medium high section but in the lower half of the section ( 7 points). For cups ranking third place, the mean ratings were 4.97 6.13 with a high-low rating difference of 1.16, which fell between medium high and low sections. For cups ranking fourth place, the mean ratings were 4.60 2.91 with a high-low rating difference of 1.69 , which fell into the medium low section. It is clear that the 4 cups were generally given low ratings in both the "visual sense" and "imagined tactile sense" tests. On a scale of 1 to 10 points, the highest mean rating was less than 9 and all ratings failed to reach the highest section, whereas the means of "imagined tactile sense" test possessed great difference in high-low ratings, suggesting that most of the test subjects were not satisfied in the visual sense test and were not willing to touch them.

Table 4. Means and standard deviations for observing simultaneously and individually in the "visual sense" test

\begin{tabular}{lcccccccc}
\hline & \multicolumn{2}{c}{ Ranking 1 } & \multicolumn{2}{c}{ Ranking 2 } & \multicolumn{2}{c}{ Ranking 3 } & \multicolumn{2}{c}{ Ranking 4 } \\
\cline { 2 - 9 } & mean & SD & mean & SD & mean & SD & mean & SD \\
\hline Cup1 & 8.50 & 0.76 & 6.00 & 1.73 & 5.58 & 1.75 & 3.68 & 1.80 \\
Cup2 & 7.73 & 1.65 & 6.56 & 2.02 & 5.36 & 1.52 & 3.59 & 1.59 \\
Cup3 & 7.79 & 1.65 & 6.36 & 1.79 & 5.24 & 1.80 & 3.00 & 1.83 \\
Cup4 & 8.20 & 1.28 & 6.68 & 1.52 & 5.00 & 1.79 & 4.24 & 1.59 \\
\hline
\end{tabular}

Table 5. Means and standard deviations for observing simultaneously and individually in the "imagined tactile sense" test

\begin{tabular}{lcccccccc}
\hline & \multicolumn{2}{c}{ Ranking 1 } & \multicolumn{2}{c}{ Ranking 2 } & \multicolumn{2}{c}{ Ranking 3 } & \multicolumn{2}{c}{ Ranking 4 } \\
\cline { 2 - 9 } & mean & SD & mean & SD & mean & SD & mean & SD \\
\hline Cup1 & 8.64 & 0.84 & 6.40 & 1.11 & 5.45 & 2.02 & 2.91 & 1.08 \\
Cup2 & 8.00 & 1.36 & 6.05 & 1.88 & 5.06 & 1.43 & 4.60 & 2.12 \\
Cup3 & 6.88 & 2.32 & 6.50 & 1.60 & 6.13 & 1.73 & 3.88 & 1.45 \\
Cup4 & 8.25 & 1.53 & 6.13 & 2.39 & 4.97 & 1.89 & 4.00 & 2.06 \\
\hline
\end{tabular}




\section{Conclusion}

For a consumer to accept a product, the first stage of preference judgment is to see and touch, yet most products originates from the creativity of designers, products are rarely produced due to the preference of general consumers. A set of cups each with different forms stands out significantly from other conventional cups in terms of creativity. Thus, the design concept to explore these cups is: "This set consists of four different shaped cups, allowing your friends and family to pick their own favorites!"

Questionnaires were used to analyze visual preference and to investigate whether if the imagined tactile sense analysis exist any difference. After four stages of tests and three phases of analysis, it was found that neither visual nor imagined tactile senses produced the anticipated outcomes. In the data where cups 2 and 3 were selected as first place, the preference of cup 2 was far greater than tactile expectation than visual sense, whereas it was the opposite for cup 3 as the preference to it was far greater in visual sense than imagined tactile sense; others that ranked from second to fourth place showed consistence of gradual decline for both visual and imagined tactile sense. Moreover, as to cup 1 and cup 4, the rankings from the first to fourth place consistently decreased as well. Interestingly, of the four cups, cup 1 had the greatest difference, while the same could not be said for the other three. Difference was observed in cup 2 and cup 3 , yet because the research only employed questionnaires to analyze the preference of visual and imagined tactile senses, hence, the study was unable to be determined the reason of the affect, this interesting finding may support subsequent studies.

As we take the visual and imagined tactile senses ranking data of the four cups, which is ranked from first to fourth place, and each added up the 1 to 10 ratings, result shows decreasing consistency as expected. However, after the calculation of each mean by sequence, the means of rankings 1 through 4 all fell within the medium high section between 8 to 6 points and the medium low section between 5 to 3 points, indicating the examinees' satisfaction difference towards the cup set was not significant, the median outcome suggests that none of the test subjects were very satisfied or very unsatisfied with any of the cups. To analyze the 1 to 10 point rating from the standard deviation point of view, the standard deviation of each group was located between 1 and 3 with 0.76 being the smallest and closest value to 1 , indicating that the ratings given by the test subjects for the products revealed great difference. This suggested two possible results: 1 . there was significant preference difference between observing the cups simultaneously and individually; and 2. there was significant satisfaction difference between the test subjects regarding the cups. Due to the fact that the questionnaire data could not provide a valid outcome, thus, this study can only provide the questionnaires for future studies to employ and achieve a more comprehensive analysis concerning consumers' preference.

Personalized products are the trend of design, a set of products with different shapes is anticipated to create novelty for consumers and to achieve a high level of preference, yet our experiment indicates that the product fails to fulfill its purpose. This issue is still worth investigating regarding product research; the results of this study can provide products which are in development a better understanding of consumer perception and a foundation for future studies. 


\section{References}

1. Chang, C.H.: Current Psychology. In: Tung Hua Book, Taipei, Taiwan (1995)

2. Chang, C.C.: Perceptual Factors Underlying Users' Image Perception toward Product Form. Doctoral thesis. National Chiao Tung University, Hsinchu, Taiwan (2000)

3. Jan, R.H.: The Corresponding Relationship between Product Image and Form Features. Master's thesis. Ming Chuan University, Taoyuan, Taiwan (2004)

4. Lin, B.L.: A Stardy on the Visual and Tactile Image of Form-Taking the Shape of Plastic Bottles as Examples. Master' s thesis, Ming Chuan University, Taoyuan, Taiwan (2002)

5. Lin, R.T.: Cultural creativity added design value. Art Appreciation 1(7), 26-32 (2005)

6. Tsai, C.Y.: A study on the influence of the form and the texture on product image through visual and tactile perception. Master's thesis, National Yunlin University of Science \& Technology, Yunlin, Taiwan(2004)

7. Yang, T.L., Ho, C.M., Luh, D.B.: Gestalt-oriented approach to form creation. Journal of Design 16(4), 19-34 (2011) 